\title{
Temporal variations in the Sun's rotational kinetic energy
}

\author{
H. M. Antia ${ }^{1}$, S. M. Chitre ${ }^{2}$, and D. O. Gough ${ }^{3}$ \\ 1 Tata Institute of Fundamental Research, Homi Bhabha Road, Mumbai 400005, India \\ e-mail: antia@tifr.res.in \\ 2 Centre for Basic Sciences, University of Mumbai, Mumbai 400098, India, and Institute of Astronomy, University of Cambridge, \\ Madingley Road, Cambridge CB3 OHA, UK \\ 3 Institute of Astronomy, University of Cambridge, Madingley Road, Cambridge CB3 0HA, and Department of Applied Mathematics \\ and Theoretical Physics, Centre for Mathematical Sciences, Wilberfore Road, Cambridge CB3 OWA, UK
}

Received 3 July 2007 / Accepted 31 October 2007

\begin{abstract}
Aims. We study the variation of the angular momentum and the rotational kinetic energy of the Sun, and associated variations in the gravitational multipole moments, on a timescale of the solar cycle.

Methods. These quantities are determined by inverting helioseismic rotational splitting data obtained by the Global Oscillation Network Group and by the Michelson Doppler Imager on the Solar and Heliospheric Observatory.

Results. The temporal variation in angular momentum and kinetic energy at high latitudes $(>\pi / 4)$ through the convection zone is positively correlated with the level of solar activity, whereas at low latitudes it is anticorrelated, except in the top $10 \%$ by radius where both are correlated positively.

Conclusions. The helioseismic data imply significant temporal variation in the angular momentum and the rotational kinetic energy, and in the gravitational multipole moments. The properties of that variation will help constrain dynamical theories of the solar cycle.
\end{abstract}

Key words. Sun: interior - Sun: rotation - Sun: activity - Sun: helioseismology - Sun: magnetic fields - Sun: oscillations

\section{Introduction}

Helioseismic data from the Global Oscillation Network Group (GONG) and the Michelson Doppler Imager (MDI) have been used to infer the rotation rate in the solar interior (Thompson et al. 1996; Schou et al. 1998). The accumulation of these data over an interval of about 11 years has made it possible to study solar-cycle-related temporal variations. Previous studies have revealed a distinct pattern of bands of faster and slower than average rotation (Howe et al. 2000a; Antia \& Basu 2000), which at low latitudes move towards the equator, and at high latitudes move towards the poles (Antia \& Basu 2001). This pattern, which is similar to the torsional oscillations observed at the solar surface (Howard \& LaBonte 1980), has been well studied, and it has been found to penetrate through much of the body of the convection zone (Vorontsov et al. 2002; Basu \& Antia 2003; Howe et al. 2005, 2006). Below the convection zone, however, there is no generally accepted unambiguous detection of significant temporal variation in the rotation, although Howe et al. (2000b, 2007) have reported a periodic variation near the equator between $r=0.65 R_{\odot}$ and $r=0.75 R_{\odot}$ in the early years of the last sunspot cycle with a period of 1.3 years, and Gough (2007) has reported that there is evidence that the oscillation penetrates even more deeply. Other studies (e.g., Antia \& Basu 2000; Basu $\&$ Antia 2003) have not confirmed such a variation.

A direct consequence of temporal variation in the angular velocity is that global quantities such as angular momentum and kinetic energy of rotation are also liable to exhibit variation with time (cf., Komm et al. 2003). Because of the steep increase in density with depth, the major contribution to these global quantities is naturally likely to arise from the deeper layers, where a temporal variation in rotation has not been determined reliably; and as a result, it is difficult to draw any firm conclusion about the global variation of angular momentum and kinetic energy. However, following Komm et al. (2003), we may study the contributions from different layers within the convection zone. We hope that such a study will shed some light on the rotational dynamics and its role in driving the activity cycle. Indeed, the study we present here is very similar to that of Komm et al. (2003), although we are now able to extend it over an entire sunspot cycle. Apart from angular momentum and kinetic energy, we address also the temporal variation of the gravitational quadrupole and higher-order multipole moments due to the distortion in the solar figure induced by the known rotation.

In this work we concentrate principally on temporal variations in angular momentum and rotational kinetic energy in different regions in the solar convection zone, and compare their variation with the solar activity. We describe in Sect. 2 the basic technique and the data used in the study; the main results are described in Sect. 3. In Sect. 4 we present the variations in the multipole moments of the external gravitational potential. We draw our conclusions in Sect. 5.

\section{The data and their analysis}

We use the data from GONG (Hill et al. 1996) and SOI/MDI (Schou 1999). Each data set consists of mean frequencies of different $(n, l)$ multiplets, and the corresponding splitting coefficients. We use 110 temporally overlapping data sets from GONG, each covering a period of 108 days, starting from 1995 May 7 and ending on 2006 May 20, each set being displaced by 36 days from its predecessor. The MDI data consist of 49 contiguous data sets, each covering a period of 72 days, starting from 1996 May 1 and ending on 2006 May 20. These data 
cover the rising phase of the cycle 23 and most of the descending phase as well. We use a 2D Regularized Least-Squares (RLS) inversion technique in the manner adopted by Antia et al. (1998) to infer the angular velocity in the solar interior from each of the available data sets.

To study the temporal variation in the angular velocity we look at the residuals obtained by subtracting from the angular velocity at any given time its temporal mean $\Omega_{0}(r, \theta)$

$\delta \Omega(r, \theta, t)=\Omega(r, \theta, t)-\Omega_{0}(r, \theta)$,

with respect to the spherical polar coordinates $(r, \theta)$, where $t$ is time. The averaging to obtain $\Omega_{0}$ is over the entire time interval within which the data are available. Both the temporal mean and the residuals were inferred separately from GONG and MDI data. The residuals, which essentially define the temporally varying component of the angular velocity, were used (together with the means) to calculate the variation with epoch in the kinetic energy and angular momentum in different regions of the Sun. For example, the change in angular momentum between spherical surfaces at radii $r_{1}$ and $r_{2}$ in the Sun can be written

$\delta J\left(t ; r_{1}, r_{2}\right)=2 \pi \int_{r_{1}}^{r_{2}} \rho r^{4} \mathrm{~d} r \int_{0}^{\pi} \delta \Omega(r, \theta, t) \sin ^{3} \theta \mathrm{d} \theta$,

where $\rho(r)$ is the density. The integrand in the outer integral defines the contribution to the angular momentum per unit radius from an infinitesimal spherical shell around radius $r$, namely $\partial \delta J / \partial r:=\partial \delta J /\left.\partial r_{2}\right|_{r_{2}=r}$. Similarly, we can also calculate the temporal variation in the kinetic energy of rotation from the expression

$\delta T\left(t ; r_{1}, r_{2}\right) \simeq 2 \pi \int_{r_{1}}^{r_{2}} \rho r^{4} \mathrm{~d} r \int_{0}^{\pi} \Omega_{0}(r, \theta) \delta \Omega(r, \theta, t) \sin ^{3} \theta \mathrm{d} \theta$.

In evaluating expressions (2) and (3) we have adopted the density $\rho(r)$ from a standard solar model.

In addition to integrating over the entire latitude range we can consider also contributions from different latitude intervals. The observed variation in angular velocity exhibits different behaviour in the low- and high-latitude regions. At low latitudes the bands of more rapidly and more slowly rotating fluid migrate towards the equator, while at high latitudes the bands move polewards (Antia \& Basu 2001; Vorontsov et al. 2002; Howe et al. 2005; Basu \& Antia 2006). We therefore consider the contribution to the integral separately over the low latitudes $(\leq \pi / 4)$ and high latitudes $(\geq \pi / 4)$ to see how they compare with the corresponding global quantities.

The integrands in Eqs. (2) and (3) are essentially the same, save for the factor $\Omega_{0}$ in Eq. (3). Therefore because the angular variation of $\Omega_{0}$ is substantial only in the polar regions where the factor $\sin ^{3} \theta$ is quite small, the temporal variations $\delta J$ and $\delta T$ are very similar. Consequently, in most of the following discussion we consider explicitly only the temporal variation in the kinetic energy. We compare it with a solar activity index, for which we use the radio flux at $10.7 \mathrm{~cm}$ as a proxy.

Apart from kinetic energy and angular momentum, it is possible to compute the gravitational quadrupole and higherorder multipole moments of the Sun resulting from the centrifugal force (e.g. Schwarzschild 1947; Sweet 1950; Gough 1981, 1982; Ulrich \& Hawkins 1981; Pijpers 1998; Antia et al. 2000; Roxburgh 2001; Mecheri et al. 2004). The gravitational potential $\Phi(r, \theta)$ outside the Sun can be written

$\Phi(r, \theta, t)=-\frac{G M_{\odot}}{r}\left[1-\sum_{k=1}^{\infty}\left(\frac{R_{\odot}}{r}\right)^{2 k} J_{2 k}(t) P_{2 k}(\cos \theta)\right]$,

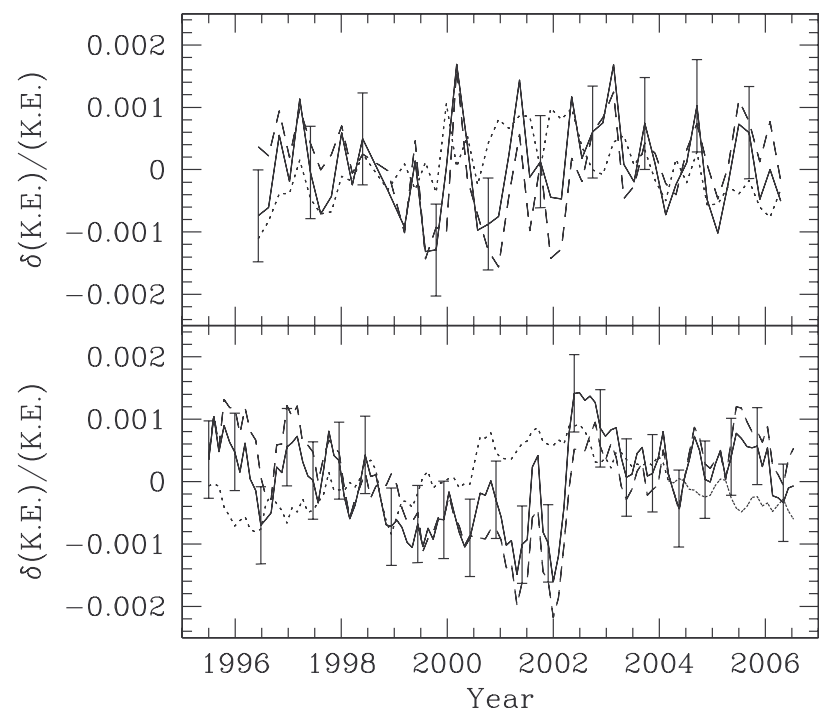

Fig. 1. Temporal variation in rotational kinetic energy throughout the convection zone. The continuous lines join values of the total kinetic energy (in units of the temporal mean) in the convection zone at different epochs; the dashed and dotted lines respectively join corresponding contributions from the low-latitude $(\leq \pi / 4)$ and high-latitude $(\geq \pi / 4)$ regions. For clarity, only a few representative error bars are shown. The values in the upper panel were obtained from the MDI data, the values in the lower panel from the GONG data.

where $J_{2 k}$ are the dimensionless multipole moments and $P_{2 k}$ are Legendre polynomials of degree $2 k$. We attempt to determine the temporal variations in these quantities.

\section{Results}

We show in Fig. 1 the temporal variation of the rotational kinetic energy of the entire convection zone, together with the contributions from the high- and low-latitude regions. The upper panel was obtained from the MDI data, the lower panel from the GONG. An oscillatory variation with about an 11-year period is evident, although there is some difference between the values inferred from the MDI and the GONG data.

Figure 2 depicts the temporal variation in rotational kinetic energy in different regions of the Sun obtained using the GONG data. The magnitude of the absolute variation increases with depth, largely on account of increasing mass density. In order to get a more uniform comparison amongst different depths, the variation relative to the mean value of the kinetic energy in each layer is plotted; then the amplitudes are rather similar. The contributions from low latitudes $(\theta>\pi / 4)$ and high latitudes $(\theta<\pi / 4)$ are also plotted. The relative magnitude of these variations is also taken with respect to the mean kinetic energy in the entire latitude range. In all cases these variations are similar in magnitude. We note that the variation in angular velocity is substantially greater at high latitudes than it is near the equator. The effect of that on the variation of the rotational kinetic energy and angular momentum is partly compensated by the fact that the high-latitude regions are closer to the rotation axis, and contribute less to the moment of inertia, a property which is reflected by the factor $\sin ^{3} \theta$ in the integrands in Eqs. (2) and (3), which on its own integrates to $5 / 6 \sqrt{2} \approx 0.589$ and $2 / 3-5 / 6 \sqrt{2}=0.077$ in the low- and high-latitude regions respectively.

At the bottom of Fig. 2 is plotted the $10.7 \mathrm{~cm}$ radio flux, which is an indicator of solar activity. It is evident that in the outer layers $\left(r \gtrsim 0.9 R_{\odot}\right)$ of the Sun the kinetic energy is greatest 


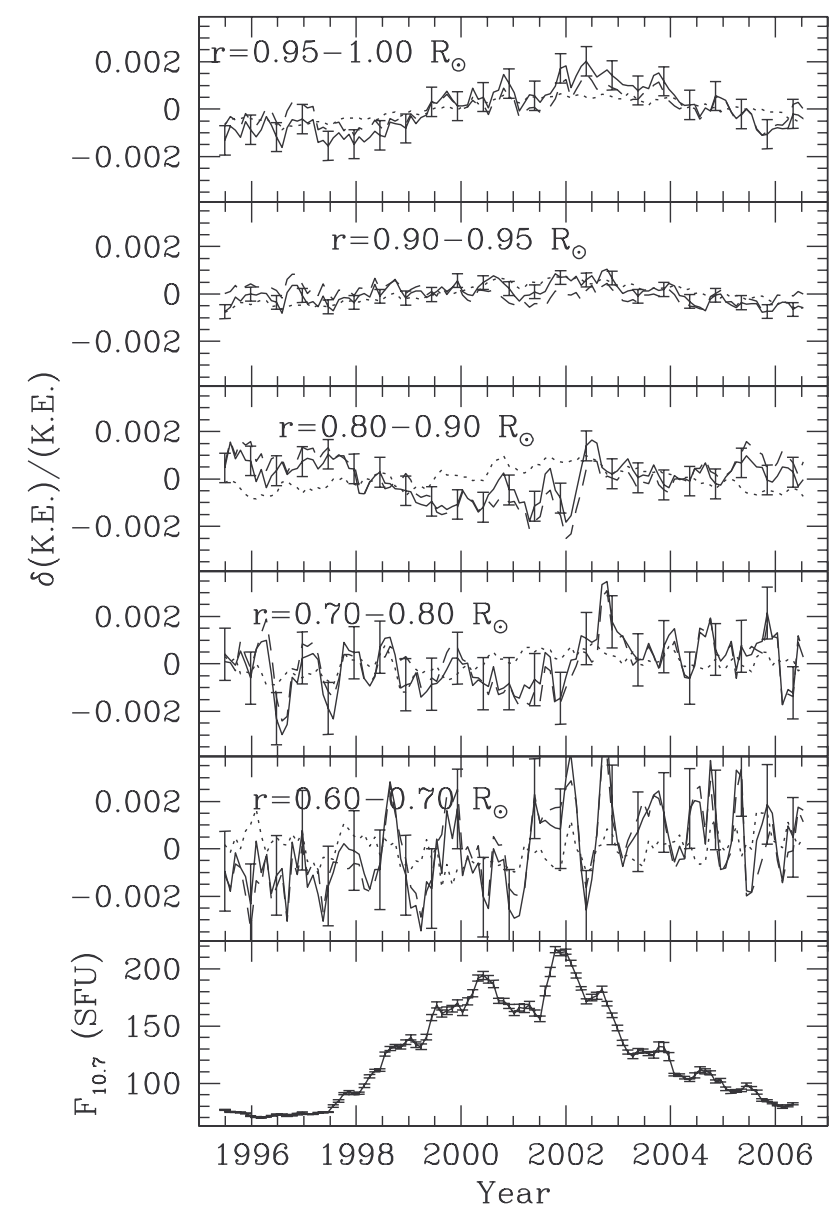

Fig. 2. Relative variation with time of the rotational kinetic energy in different regions (indicated in each panel) of the Sun, obtained from the GONG data. The continuous lines indicate the variation over entire spherical shells, and the dashed and dotted lines respectively indicate the variation in the low- $(\leq \pi / 4)$ and high-latitude $(\geq \pi / 4)$ regions. For clarity, only a few representative error bars are shown. The lowest panel depicts the variation in solar activity as measured by the $10.7 \mathrm{~cm}$ radio flux over the same time interval.

at epochs of greatest activity, both in the low-latitude and in the high-latitude regions. At intermediate depths $\left(0.7 \lessgtr r / R_{\odot} \lesssim 0.9\right)$ the high-latitude correlation with activity persists, but at low latitudes the correlation is reversed. This trend is seen more clearly in Fig. 3, in which the correlation coefficients are plotted against radius, both for entire spherical shells and for the high- and lowlatitude regions. The amplitude of the variation is the greater in the low-latitude region, so the behaviour there is an indicator of the behaviour of the kinetic energy, and the angular momentum, over the entire spherical shell. At the greatest depths it is more difficult to see the trends in Fig. 2 because the uncertainties in the inversions are comparable with the trends themselves. However, the covariance of the kinetic energy with the activity formally changes sign at $r \simeq 0.7 R_{\odot}$, at both low and high latitudes, beneath which the low-latitude kinetic energy variation is again positively correlated with activity and the high-latitude variation is negatively correlated. Again, this behaviour is visible more clearly in Fig. 3.

These results are consistent with those of Komm et al. (2003), who considered the angular-momentum variations over entire spherical shells. The relative variations in angular momentum and rotational kinetic energy, and also, of course, the angular velocity, are of order $0.1 \%$. Given that the total angular

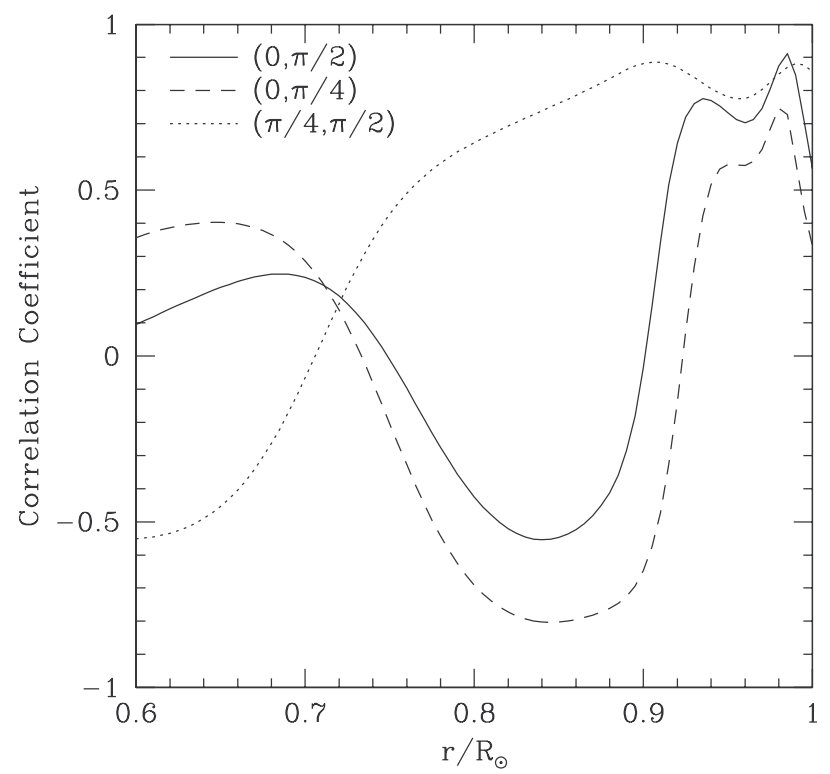

Fig. 3. Correlation coefficients between the temporal variation in kinetic energy of rotation obtained using GONG data and the $10.7 \mathrm{~cm}$ radio flux, plotted as a function of radius. The continuous curve is the coefficient for an entire spherical shell; the dashed and dotted curves are respectively the correlation coefficients for the low-latitude $(\leq \pi / 4)$ and high-latitude $(\geq \pi / 4)$ regions.

momentum and rotational kinetic energy of the convection zone are $J_{\mathrm{c}} \simeq 2.8 \times 10^{47} \mathrm{~g} \mathrm{~cm}^{2} \mathrm{~s}^{-1}$ and $T_{\mathrm{c}} \simeq 4.0 \times 10^{41} \mathrm{erg}$, this implies that the amplitudes of their variations are about $1 \times$ $10^{44} \mathrm{~g} \mathrm{~cm}^{2} \mathrm{~s}^{-1}$ and $3 \times 10^{38}$ erg respectively.

The change in sign at $r \simeq 0.9 R_{\odot}$ of the correlation of angular momentum with activity at low latitudes could be associated with the upward migration of the zonal flow pattern reported by Basu \& Antia (2003): because the migration timescale from intermediate depths is about half a cycle period, the flow pattern at intermediate depths is anticorrelated with that at the surface. At high latitudes, the angular-momentum variation is positively correlated with surface activity throughout the convection zone. Beneath the convection zone the correlation appears to be weaker, possibly as a result of the greater uncertainties in the inferred angular velocity, and may not be significant.

To calculate the amplitude of the solar-cycle variation in kinetic energy, we fit a sinusoid with a period of 11 years to the observed kinetic energy. Since the power spectra at most depths show a peak at frequencies corresponding to approximately this value, we may be justified in looking for such variation. The resulting amplitude of the relative variation is plotted against radius in Fig. 4. The dips in the curves occur near the depths at which the correlation coefficient changes sign (see Fig. 3). At these depths the amplitude might be expected to be small. Beneath the convection zone the rise in amplitude with depth might be merely a reflection of the increasing errors in the inferred angular velocity, and is therefore not to be trusted; this is suggested partly by the low correlation coefficients evident in Fig. 3.

We conclude this discussion by once again comparing inferences from GONG and MDI. Figure 5 depicts the correlation coefficients between the $10.7 \mathrm{~cm}$ radio flux and the spherically averaged rotational kinetic energy and angular momentum obtained from the two data sets. It is evident that there is very little difference between the coefficients for rotational kinetic energy and angular momentum. There is some discrepancy between the 


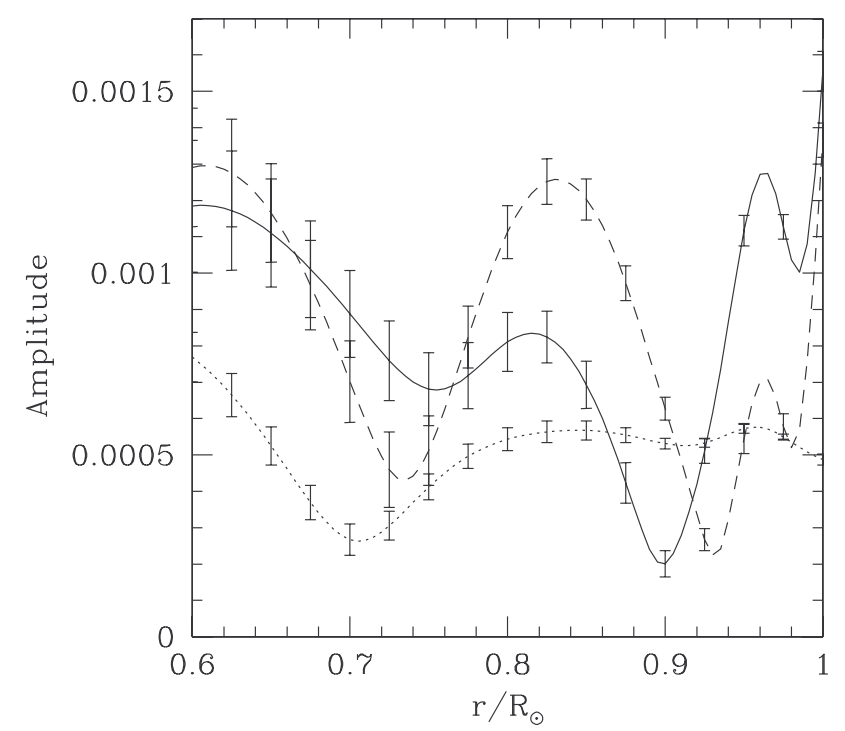

Fig. 4. Amplitude of relative variation in rotational kinetic energy, plotted against radius, obtained by fitting a sinusoid with a period of 11 years to the inferences from GONG data. The continuous curve represents the entire spherical shell, the dashed and dotted curves respectively represent the low- $(\leq \pi / 4)$ and high- $(\geq \pi / 4)$ latitude regions.

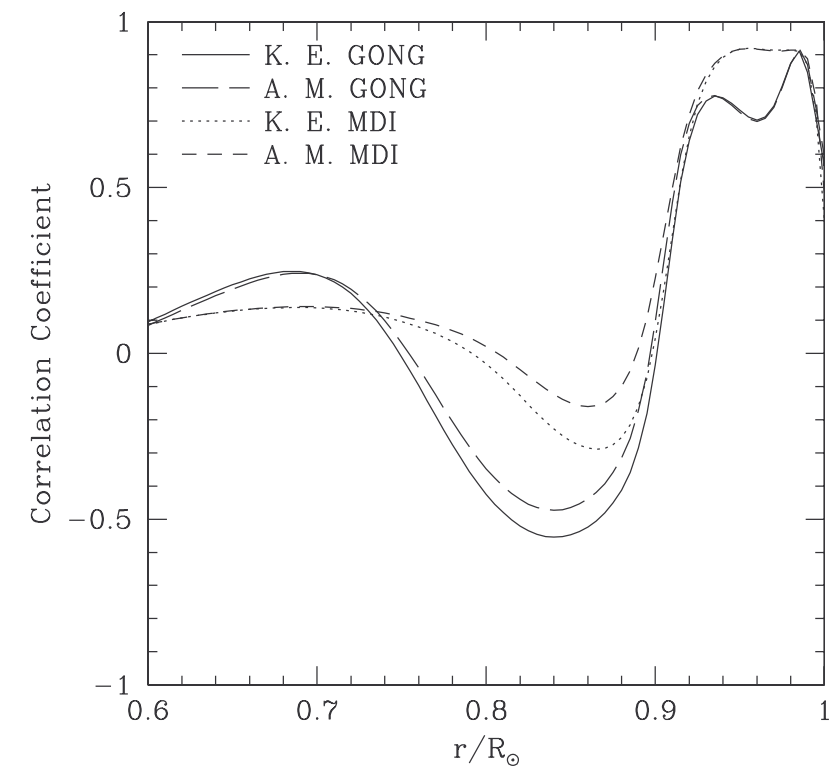

Fig. 5. Correlation coefficient between the the $10.7 \mathrm{~cm}$ radio flux and the kinetic energy and angular momentum, integrated over entire spherical shells, plotted against radius. The solid and long-dashed curves are kinetic energy and angular momentum inferred from GONG data, the dotted and short-dashed curves are from MDI data.

inferences from GONG and MDI in the lower part of the convection zone, a discrepancy similar to one that has been found also in other works (e.g., Schou et al. 2002). The reason for that discrepancy is not understood. Nevertheless, it does not affect the principal conclusions of this paper.

Howe et al. (2000b) and Komm et al. (2003) have reported a 1.3-year periodicity in the temporal variation of the angular velocity at low latitudes in the vicinity of the tachocline. Here we examine this finding further, by seeking such a periodicity in the angular momentum or kinetic energy. We calculate the discrete Fourier transforms of the results illustrated in Fig. 2 to see whether there is a significant peak in the power spectra around

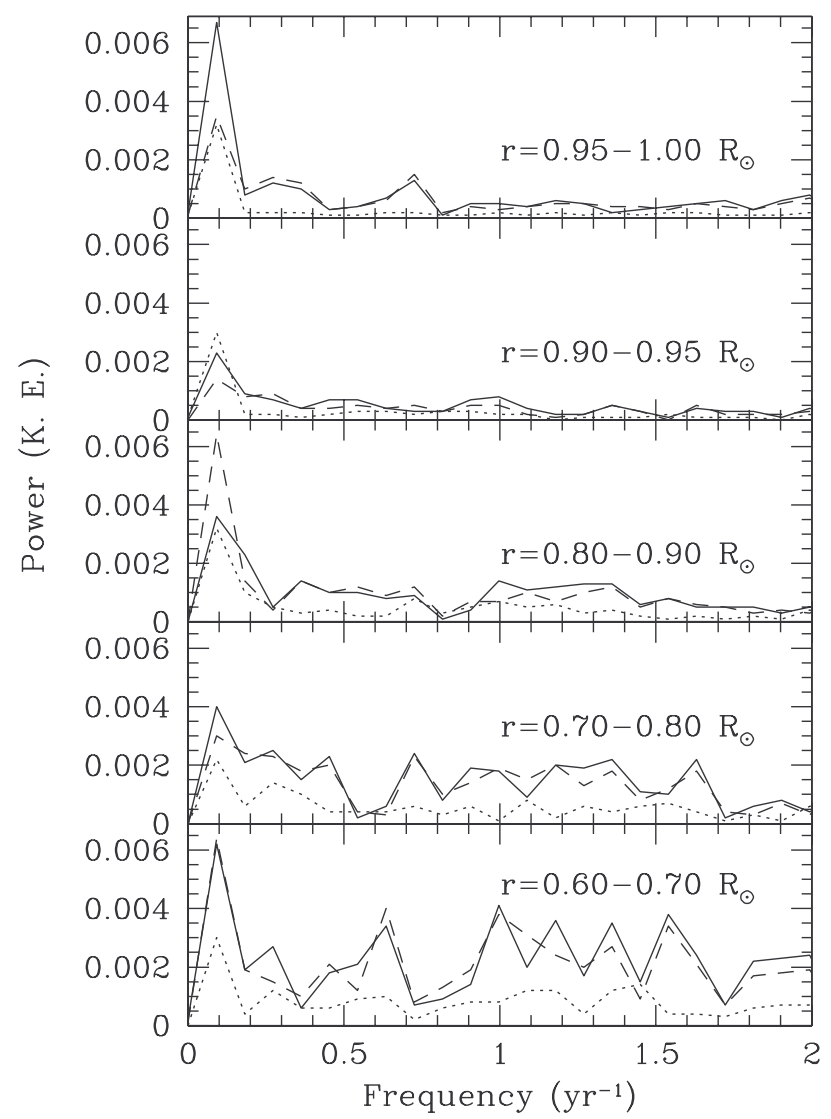

Fig. 6. Power spectrum of temporal variation in rotational kinetic energy in different depth ranges (as marked in each panel). The solid lines represent the entire spherical shell, the dashed and dotted lines represent the low- $(\leq \pi / 4)$ and high- $(\geq \pi / 4)$ latitude regions, respectively. The seismic data were obtained from GONG; the results from MDI are similar, and have been omitted for concision.

a frequency of $0.75 \mathrm{yr}^{-1}$. The outcome is presented in Fig. 6 . Evidently, no such peak is apparent. The power spectra in the vicinity of the tachocline appear to contain no particularly significant peak, although those in the upper layers of the convection zone do contain an obvious peak in the lowest frequency bin allowed in the discrete Fourier transform: this corresponds to the solar-cycle variation. We have looked at many different depth and latitude ranges; the results are all similar to those shown in the figure, irrespective of whether the seismic data employed were from GONG or from MDI. Therefore, apart from a possible periodicity characteristic of solar-cycle variation, we find no evidence for periodicity at any depth. For illustrative purposes, we present in Fig. 7 the temporal variation in the low-latitude region of the tachocline $\left(0.70 R_{\odot} \leq r \leq 0.74 R_{\odot}\right)$ in both rotational kinetic energy and angular momentum, inferred from MDI as well as GONG data. This is the region in which Howe et al. (2000b) and Komm et al. (2003) found the greatest amplitude of the 1.3-year oscillations. It is clear from the figure that there is reasonable agreement between GONG and MDI data, and that the variation in angular momentum is similar to that in the rotational kinetic energy. However, no significant temporal variation is evident; in particular, there is no very clear sign of a 1.3-year oscillation. 
Table 1. Temporal variation in multipole moments.

\begin{tabular}{ccccccc}
\hline \hline$k$ & \multirow{2}{*}{$\left\langle J_{k}\right\rangle$} & MDI & Phase (years) & \multicolumn{2}{c}{$\left|\delta J_{k}\right|$} \\
& \multicolumn{1}{c}{ GONG } & \multicolumn{1}{c}{ MDN } & MDI & GONG & MDI \\
\hline 2 & $(2.18 \pm 0.005) \times 10^{-7}$ & $(2.22 \pm 0.009) \times 10^{-7}$ & 4.1 & 8.7 & $1.0 \times 10^{-10}$ & $9.5 \times 10^{-11}$ \\
4 & $-(4.70 \pm 0.06) \times 10^{-9}$ & $-(3.97 \pm 0.09) \times 10^{-9}$ & 1.4 & 0.1 & $4.9 \times 10^{-11}$ & $7.9 \times 10^{-11}$ \\
6 & $-(2.4 \pm 0.2) \times 10^{-10}$ & $-(0.8 \pm 0.3) \times 10^{-10}$ & 7.3 & 10.7 & $4.8 \times 10^{-12}$ & $1.3 \times 10^{-11}$ \\
8 & $-(0.8 \pm 0.6) \times 10^{-11}$ & $(1.1 \pm 0.9) \times 10^{-11}$ & 4.5 & 3.6 & $1.6 \times 10^{-11}$ & $1.7 \times 10^{-11}$ \\
10 & $(7.1 \pm 4.0) \times 10^{-12}$ & $(7.4 \pm 4.0) \times 10^{-12}$ & 1.6 & 1.4 & $7.3 \times 10^{-12}$ & $7.8 \times 10^{-12}$ \\
12 & $-(1.9 \pm 2.4) \times 10^{-12}$ & $-(1.8 \pm 2.0) \times 10^{-12}$ & 6.8 & 6.9 & $3.2 \times 10^{-12}$ & $2.6 \times 10^{-12}$ \\
\hline
\end{tabular}

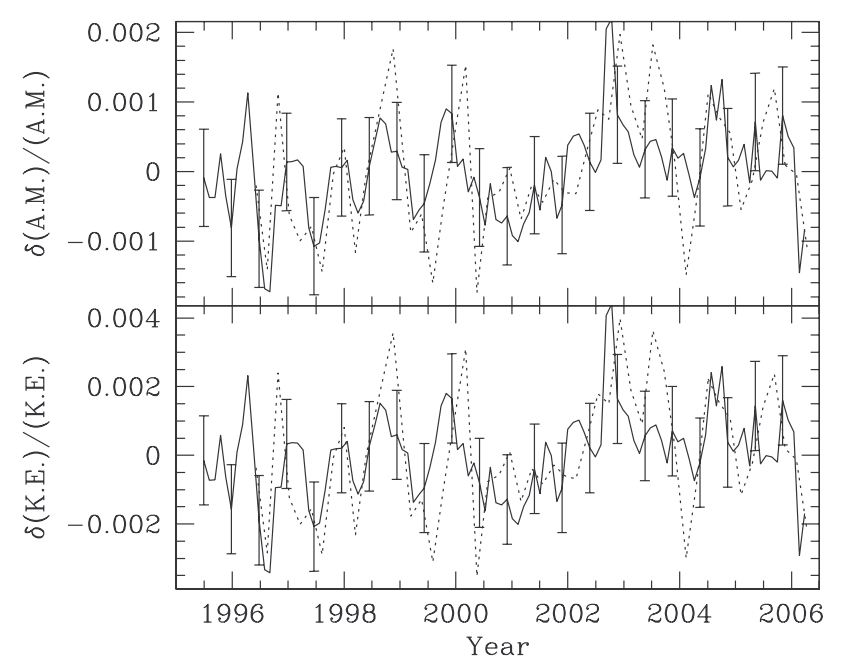

Fig. 7. Temporal variation in rotational kinetic energy and angular momentum in the low-latitude region with $0.70 R_{\odot} \leq r \leq 0.74 R_{\odot}$. The continuous lines are the result of using GONG data, the dotted lines are from using MDI data. For clarity, only a few representative error bars are shown.

\section{Gravitational multipole moments due to internal rotation}

From our inferences of the angular velocity throughout the solar interior, it is possible to calculate the quadrupole and higherorder multipole moments of the Sun's gravitational field, and study their possible temporal variation. Figure 8 illustrates the even-order moments $J_{2}-J_{12}$ inferred from both GONG and MDI data. To ascertain whether temporal variation in these quantities is related to solar activity we have calculated the coefficients of their correlation with the $10.7 \mathrm{~cm}$ radio flux. We identify the phase of any possible solar-cycle variation by fitting to the moments sinusoids with a period of 11 years. The results are summarized in Table 1, in which are listed the temporal averages of the moments as well as the relative amplitudes and phases (in years, relative to the phase of the $10.7 \mathrm{~cm}$ radio flux) of the fitted sinusoidals. As one might expect, the quadrupole moment $J_{2}$ exhibits no noticeable temporal variation: the sinusoidal fits have very low (relative) amplitudes, and the phases inferred from the GONG and MDI data are different. There appears to be a substantial difference between the mean value of $J_{2}$ calculated from the GONG and the MDI data. This is most probably a reflection of already known differences in the splitting coefficients (Schou et al. 2002). Similar differences between GONG and MDI estimates for $J_{2}$ were found by Pijpers (1998). We note in passing that Emilio et al. (2007), using measurements by MDI, find strong variation in the figure of the Sun, which is contrary to their earlier results (Kuhn et al. 1998); however, most of the distortion from sphericity is the direct response to the centrifugal

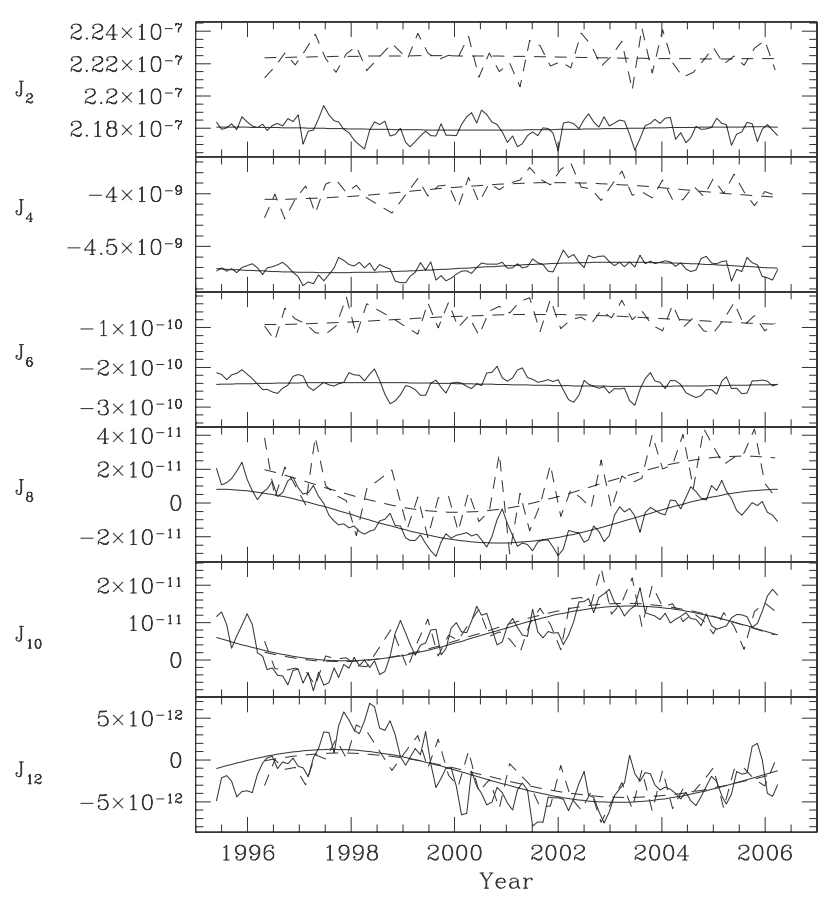

Fig. 8. Temporal variation in (even) multipole moments $J_{2}-J_{12}$. The solid lines are the results from using GONG data, dashed lines from using MDI data. In all cases a sinusoidal fit, with a period of 11 years, is also shown.

force on the rotating surface layers, and not from the asphericity of the gravitational field. The temporal variation in the distortion observed at the solar surface is certainly associated with the magnetic field, which itself is correlated with the zonal bands of alternate fast and slow rotation, and is probably confined to layers near the surface. Of course, the uncertainty in the estimate of the gravitational distortion obtained from the shape of the surface of the Sun is much greater than the uncertainty with which $J_{2}$ can be obtained from seismic measurements.

Kuhn et al. (1998) found significant temporal variation in the $P_{4}(\cos \theta)$ component of distortion at the solar surface, although their errors are much larger in this component. Accordingly, we seek a corresponding variation in $J_{4}$. It appears in Fig. 8 that there is indeed some systematic variation, for inferences from both GONG and MDI data behave similarly. However, the amplitude of the sinusoid fitted to the GONG data is only about $60 \%$ of that of the corresponding MDI sinusoid, and, moreover, the phases differ by about 1.3 years. The correlation coefficients between the inferred values of $J_{4}$ and the $10.7 \mathrm{~cm}$ radio flux are only 0.29 and 0.51 for GONG and MDI data respectively, although these quite low values appear to be a consequence mainly of the phase difference. However, the results do suggest that $J_{4}$ may undergo temporal variation with an amplitude of about 
$10^{-10}$, which is $2 \%$ of the mean, and that this variation is correlated with solar activity. The variation is much less than what Kuhn et al. (1998) found in the $P_{4}(\cos \theta)$ component of the distortion from sphericity of the shape of the Sun's surface; the difference could be in part a product of large errors in the distortion observed, but it is likely to be due mainly to the direct effect of the centrifugal force, and also the magnetic field and accompanying velocity and temperature variations associated with solar activity in the low-density surface layers, which influence the figure but hardly modify the gravitational field, even though the kernels for the high-order multipole moments are more concentrated near the surface. The mean value of $J_{4}$ in Table 1 is close to that found by Roxburgh (2001) and Mecheri et al. (2004), who used rough approximations to the seismically inferred rotation rate.

The variations in $J_{6}$ that we have inferred from the GONG and MDI data do not agree: whereas the MDI data suggest a clear variation which is correlated with solar activity, the GONG data reveal none. The mean values of $J_{6}$ differ by a factor 3 , although they are at least of the same order as that found by Roxburgh (2001).

The variation in $J_{8}$ is anticorrelated with solar activity, and now the variations inferred from both GONG and MDI data look similar. However, the mean values do not even have the same sign. With $J_{10}$ and $J_{12}$ there is good agreement between GONG and MDI. The magnitudes of $J_{8}$ and higher-order moments are comparable with their temporal variations, although we hasten to add that they are comparable also with the estimated 1- $\sigma$ errors in the individual estimates. Therefore they may not be significant. But we have presented them because they do tend to show definite temporal trends which are similar in both the GONG and the MDI inferences. Higher-order multipole moments also exhibit variation, but we do not present them because their reliability is certainly questionable.

We point out that the contribution from rotation to each multipole moment can be expressed as a bilinear function of the odd degeneracy splitting coefficients of the seismic modes, a function which depends also on the density $\rho(r)$. Therefore, because the density of the same reference solar model was used to compute all the moments, whether the seismic data used were from GONG or MDI, all the differences amongst the inferences must have their origin solely in the differences amongst the measured splitting coefficients.

\section{Summary and conclusions}

We have studied the temporal variation in the rotational kinetic energy and angular momentum of the Sun in different regions of the convection zone. We find that in the outer layers $\left(r \gtrsim 0.9 R_{\odot}\right)$ the variation is well correlated with solar activity. At high latitudes this correlation continues throughout the convection zone, whereas at low latitudes (and also when integrated over all latitudes), there is a transition to anticorrelation around $r=0.9 R_{\odot}$. Through the bulk of the convection zone the high latitudes seem to be rotating faster during the solar activity maximum, and the equatorial regions rotate more slowly. It is difficult to draw any definite conclusions about the temporal variation below the convection zone, however, because there the errors are large.

The amplitude of the temporal variation in the rotational kinetic energy integrated over the entire convection zone is of order of $3 \times 10^{38} \mathrm{erg}$. This implies a rate of variation whose amplitude is about $5 \times 10^{30} \mathrm{erg} \mathrm{s}^{-1}$. It is not clear if this variation is compensated for by opposite variation in deeper layers or whether it represents exchange of energy between rotation and
Table 2. Rotational kinetic energy, thermal energy and the estimated rms magnetic field using Eq. (5) in different layers.

\begin{tabular}{cccc}
\hline \hline Radial extent & $\begin{array}{c}\text { Mean rot. KE } \\
(\mathrm{erg})\end{array}$ & $\begin{array}{c}\text { rms } B \\
(\mathrm{G})\end{array}$ & $\begin{array}{c}\text { Thermal energy } \\
(\mathrm{erg})\end{array}$ \\
\hline $0.99<r / R_{\odot}<1.00$ & $5.3 \times 10^{37}$ & 250 & $6.2 \times 10^{40}$ \\
$0.95<r / R_{\odot}<0.99$ & $6.5 \times 10^{39}$ & 1400 & $2.4 \times 10^{43}$ \\
$0.85<r / R_{\odot}<0.95$ & $9.3 \times 10^{40}$ & 3700 & $1.4 \times 10^{45}$ \\
$0.75<r / R_{\odot}<0.85$ & $2.0 \times 10^{41}$ & 6100 & $7.6 \times 10^{45}$ \\
$0.65<r / R_{\odot}<0.75$ & $2.6 \times 10^{41}$ & 7900 & $2.2 \times 10^{46}$ \\
\hline
\end{tabular}

magnetic field or some other form. It is interesting to note that this value is roughly comparable to (actually about 3 times) the amplitude of the observed variation in the solar irradiance, which is about $0.04 \%$, although the correspondence might well be fortuitous. It is, however, tempting to speculate that the temporal variation in rotational kinetic energy, and the magnetic energy, has some role to play in the irradiance variation. A part of the variation in the rotational kinetic energy would be expected to be compensated by the magnetic energy stored in the solar convection zone. If that were the case at all radii, we can estimate the spatial variation of the rms magnetic field using the relation

$\frac{B^{2}}{8 \pi} 4 \pi r^{2} \delta r \approx \delta T$

The total variation $\delta T$ in $T$ over the solar cycle is about $0.002 T$, where $T\left(r_{1}, r_{2}\right)$ is the mean rotational kinetic energy in the region between radii $r_{1}$ and $r_{2}$. Using this, together with the mean kinetic energy integrated over all latitudes within several spherical shells, we list in Table 2 these admittedly very crude estimates of the magnetic field.

The gravitational quadrupole moment $J_{2}$ does not exhibit any significant temporal variation, whereas some other, higherorder, multipole moments do. The variation in $J_{4}$ is correlated with solar activity, but the amplitudes of variation seen in GONG and MDI data are somewhat different. Clear variation in $J_{8}-J_{12}$ is inferred from both GONG and MDI data, the variation in $J_{10}$ being correlated with solar activity and that in $J_{8}$ and $J_{12}$ anticorrelated. The absence of temporal variation in $J_{2}$ is consistent with an observed absence of variation in the $P_{2}(\cos \theta)$ component of distortion at the solar surface (Kuhn et al. 1998). This is to be expected, since $J_{2}$ is determined essentially by the spherically averaged component of the angular velocity, which suffers little relative variation. The higher-order multipole moments are determined also by the latitudinally varying components of the angular velocity, which are known to suffer temporal variation correlated with solar activity. Note that the predominant contribution to $J_{2}$ arises from regions near the base of the convection zone (Gough 1981; Pijpers 1998), whereas the higher-order multipole moments arise largely from contributions from outer layers of the Sun, where the temporal variation in the rotation rate is not insubstantial. Of course, the magnitude of $J_{2}$ is much larger than that of the higher multipole moments, and it is not unlikely that $J_{2}$ also has temporal variations with comparable absolute magnitude.

Acknowledgements. This work utilized data obtained by the Global Oscillation Network Group (GONG) project, managed by the National Solar Observatory, which is operated by AURA, Inc. under a cooperative agreement with the National Science Foundation. The data were acquired by instruments operated by the Big Bear Solar Observatory, High Altitude Observatory, Learmonth Solar Observatory, Udaipur Solar Observatory, Instituto de Astrofisico de Canarias, and Cerro Tololo Inter-American Observatory. This work also utilises data from the Solar Oscillations Investigation/Michelson Doppler Imager (SOI/MDI) on 
the Solar and Heliospheric Observatory (SOHO). SOHO is a project of international cooperation between ESA and NASA. We are grateful to J. ChristensenDalsgaard, T. Duvall, L. Gizon and P. Scherrer for helpful discussions and valuable comments. S.M.C. thanks DAE-BRNS for support under the Senior Scientist Scheme and is also grateful to the Institute of Astronomy, Cambridge for supporting his visits during the course of this study.

\section{References}

Antia, H. M., \& Basu, S. 2000, ApJ, 541, 442

Antia, H. M., \& Basu, S. 2001, ApJ, 559, L67

Antia, H. M., Basu, S., \& Chitre, S. M. 1998, MNRAS, 298, 543

Antia, H. M., Chitre, S. M., \& Thompson, M. J. 2000, A\&A, 360, 335

Basu, S., \& Antia, H. M. 2003, ApJ, 585, 553

Basu, S., \& Antia, H. M. 2006, in Proc. SOHO 18/GONG 2006/HELAS I workshop on Beyond the Spherical Sun, ed. K. Fletcher, \& M. J. Thompson, ESA SP-624, 128

Emilio, M., Bush, R. I., Kuhn, J., \& Scherrer, P. 2007, ApJ, 560, 161

Gough, D. O. 1981, MNRAS, 196, 731

Gough, D. O. 1982, Nature, 298, 334

Gough, D. O. 2007, in Dynamics of the Solar Tachocline, ed. D. W. Hughes, R. Rosner, \& N. O. Weiss (Cambridge University Press)
Hill, F., Stark, P. B., Stebbins, R. T., et al. 1996, Science, 272, 1292 Howard, R., \& LaBonte, B. J. 1980, ApJ, 239, L33

Howe, R., Christensen-Dalsgaard, J., Hill, F., et al. 2000a, ApJ, 533, L163

Howe, R., Christensen-Dalsgaard, J., Hill, F., et al. 2000b, Science, 287, 2456

Howe, R., Christensen-Dalsgaard, J., Hill, F., et al. 2005, ApJ, 634, 1405

Howe, R., Rempel, M., Christensen-Dalsgaard, J., et al. 2006, ApJ, 649, 1155

Howe, R., Christensen-Dalsgaard, J., Hill, F., et al. 2007, Adv. Space Res., 40, 915

Komm, R., Howe, R., Durney, B. R., \& Hill, F. 2003, ApJ, 586, 650

Kuhn, J. R., Bush, R. I., Scheick, X., \& Scherrer, P. 1998, Nature, 392, 155 Mecheri, R., Abdelatif, T., Irbah, A., Provost, J., \& Berthomieu, G. 2004, Sol. Phys., 222, 191

Pijpers, F. P. 1998, MNRAS, 297, L76

Roxburgh, I. W. 2001, A\&A, 377, 688

Schou, J. 1999, ApJ, 523, L181

Schou, J., Antia, H. M., Basu, S., et al. 1998, ApJ, 505, 390

Schou, J., Howe, R., Basu, S., et al. 2002, ApJ, 567, 1234

Schwarzschild, M. 1947, ApJ, 106, 427

Sweet, P. A. 1950, MNRAS, 110, 548

Thompson, M. J., Toomre, J., Anderson, E., et al. 1996, Science, 272, 1300

Ulrich, R. K., \& Hawkins, G. W. 1981, ApJ, 246, 985; Erratum ApJ, 249, 831

Vorontsov, S. V., Christensen-Dalsgaard, J., Schou, J., Strakhov, V. N., \& Thompson, M. J. 2002, Science, 296, 101 